\title{
Miranda
}

Revue pluridisciplinaire du monde anglophone /

Multidisciplinary peer-reviewed journal on the English-

speaking world

$20 \mid 2020$

Staging American Nights

\section{Muriel Plana, Nathalie Vincent-Arnaud, Ludovic Florin et Frédéric Sounac, dir., Théâtre musical (XXe et XXIe siècles) : Formes et représentations politiques}

\section{Pierre Degott}

\section{OpenEdition}

Journals

Édition électronique

URL : http://journals.openedition.org/miranda/23222

DOI : $10.4000 /$ miranda.23222

ISSN : 2108-6559

\section{Éditeur}

Université Toulouse - Jean Jaurès

\section{Référence électronique}

Pierre Degott, « Muriel Plana, Nathalie Vincent-Arnaud, Ludovic Florin et Frédéric Sounac, dir., Théâtre musical (XXe et XXIe siècles) : Formes et représentations politiques », Miranda [En ligne], 20 | 2020, mis en ligne le 19 mars 2020, consulté le 16 février 2021. URL : http://journals.openedition.org/miranda/ 23222 ; DOI : https://doi.org/10.4000/miranda.23222

Ce document a été généré automatiquement le 16 février 2021.

\section{cc)}

Miranda is licensed under a Creative Commons Attribution-NonCommercial-NoDerivatives 4.0

International License. 
Muriel Plana, Nathalie VincentArnaud, Ludovic Florin et Frédéric Sounac, dir., Théâtre musical (XXe et XXIe siècles) : Formes et représentations politiques

Pierre Degott

\section{RÉFÉRENCE}

Muriel Plana, Nathalie Vincent-Arnaud, Ludovic Florin et Frédéric Sounac, dir., Théâtre musical (XXe et XXIe siècles) : Formes et représentations politiques (Besançon : Presses Universitaires de Franche-Comté, 2019), 228 p, ISBN : 978-2-84867-664-7 


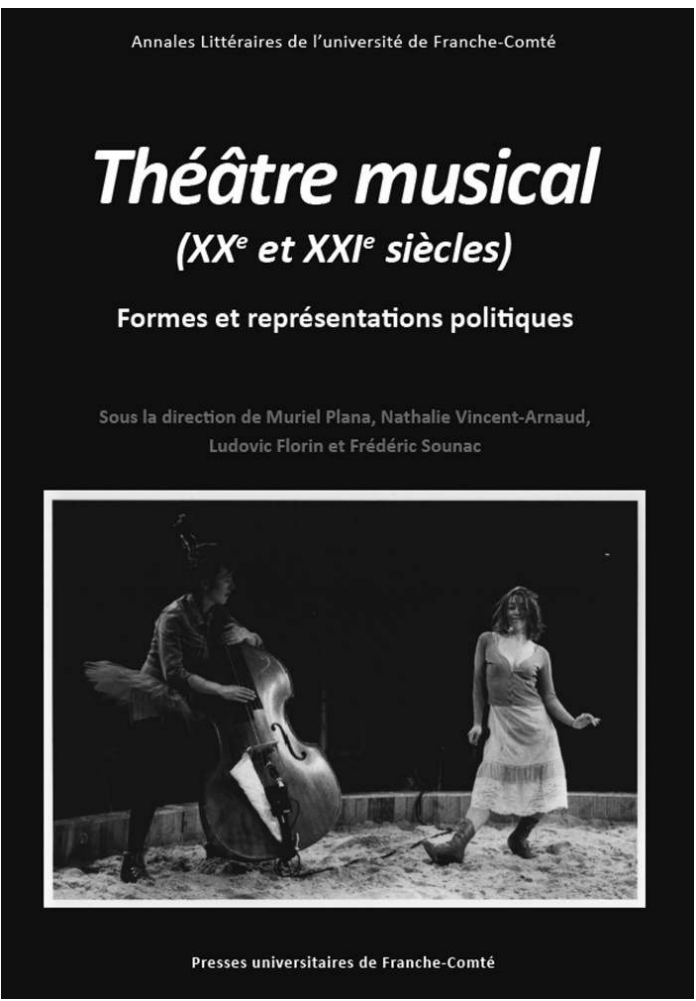

1 Difficile à définir, le genre artistique du théâtre musical continue à déconcerter autant qu'il fascine. Indéterminé sur le plan historique et chronologique, sauf à décider qu'il est né ex nihilo avec Brecht et Weill, il l'est encore plus sur le plan générique. S'agit-il, comme le rappelle Muriel Plana au cours de l'article sur lequel se clôt le volume, de musicalisation du théâtre ou plutôt de théâtralisation de la musique? On sait l'engouement actuel pour le concert théâtralisé... Quelle est l'articulation entre le théâtre musical et l'opéra, ce genre dit fusionnel qui depuis Wagner prône haut et fort le principe de la réunion des arts? De quelle manière le théâtre musical, genre qui n'exclut pas a priori des liens proches avec la comédie musicale spectaculaire commerciale (ou musical), résout-il la question éminemment problématique de la rencontre entre culture savante et culture populaire? En somme, la question revient à se demander comment il faut appréhender les diverses manifestations du phénomène de l'hybridité - et non du métissage - qui semble caractériser un genre lequel, de par la pluralité des formes qu'il revêt aujourd'hui, continue de nous échapper.

2 En dépit de l'intérêt que porte actuellement le monde académique et artistique aux formes musicothéâtrales hybrides (voir la bibliographie aux pages 217-220), les recherches sur la définition d'un théâtre musical moderne sont rares et encore balbutiantes. Le présent volume, issu de séminaires menés par l'Institut IRPALL de l'université Toulouse-Jean Jaurès, vient à point nommé proposer un certain nombre de réponses aux principales interrogations contemporaines. Remarquablement organisé et structuré, il rassemble une douzaine d'articles consacrés soit à des figures emblématiques du théâtre musical des XXe et XXIe siècles, soit à certains aspects théoriques propres à un genre artistique problématique entre tous. Chacune des contributions apporte à l'édifice en construction un regard nouveau sans pour autant figer par un excès de théorisation une forme musicothéâtrale aux contours encore fluides, qui n'a pas cessé de nous étonner. 
3 L'ouvrage démarre en toute logique avec le seul article spécifiquement consacré à une œuvre écrite par Bertolt Brecht. Le texte de Karine Saroh traite en effet de la collaboration entre le grand dramaturge allemand et le compositeur Hanns Eisler, l'ouvrage Die Maßnahme (1930). L'article rappelle les contenus didactiques, militants, sociaux et politiques de cette pièce tout en insistant, et l'on s'en réjouit, sur les aspects structurels et rhétoriques d'une œuvre musicolittéraire dont la réception et les contextes historiques auraient peut-être pu être évoqués plus fermement. Presque contemporaine de l'ouvrage de Brecht et Eisler, la pièce en un acte The Dance of Death (1933) écrite par W.H. Auden, avec une musique du compositeur Herbert Murrill, fait l'objet d'une belle étude de Pierre Longuenesse. On la retrouvera dans le numéro 20 de La Revue Musicorum. Tous les textes suivants se penchent sur des productions datant de l'après-guerre. Stefan Keym étudie ainsi deux ouvrages phares des années 1950 et 1970, l'Abstrakte Oper Nr. 1 («Opéra abstrait $\mathrm{N}^{\circ} 1 »$; 1953) du compositeur Boris Blacher (œuvre écrite sur la base d'une idée et d'un texte de son collègue Werner Egk), ainsi que le Satyricon (1973) de Bruno Maderna. Les deux ouvrages sont l'occasion de creuser le concept du Literaturoper - opéra fondé sur des textes littéraires canoniques - et d'interroger les liens indissociables qu'entretiennent les genres de l'opéra et du théâtre musical. Un des grands mérites du bel article que Jean-François Trubert consacre à l'une des toutes premières œuvres de Mauricio Kagel, Sonant (1960), est d'analyser avec finesse la manière dont cette partition a été conçue pour mettre en avant les modalités de ce que le compositeur argentin a défini comme le théâtre instrumental. Le texte analyse donc le phénomène selon lequel Kagel procède à une mise en scène de tous les ressorts visuels et gestuels - gestes déictiques, iconiques et métaphoriques - destinés à exploiter au mieux le potentiel théâtral du jeu des instrumentistes. L'article de JeanMichel Court, placé un peu plus loin dans le volume, poursuit cette réflexion à partir de l'œuvre du compositeur Dieter Schnebel, dont l'auteur étudie le souci de recourir à une nouvelle forme d'expressivité - se plaçant ainsi en porte-à-faux par rapport à la doxa boulézienne de l'époque -, notamment par la déconstruction qu'il propose du travail sur la voix ; c'est tout l'enjeu de ses compositions Maulwerke (littéralement « œuvres de gueule »; 1968-1974) pour lesquels il y a en réalité moins besoin de chanteurs que d'articulateurs. Il eût été étonnant que la figure de Luigi Nono ne soit pas évoquée dans un volume comme celui-ci. C'est ce que fait Karine Saroh dans son analyse du fameux Al gran sole carico d'amore créé à la Scala de Milan en 1975, et dont elle nous rappelle tous les contenus politiques ainsi que les stratégies mises en place pour rejeter coûte que coûte, pour un ouvrage créé ironiquement dans le saint des saints de l'art lyrique, la modalité fusionnelle de la relation entre les arts. Consacré à des exemples empruntés à Georges Aperghis et à Karlheinz Stockhausen - Machinations (2000) et Avis de tempête (2004) pour le premier, Mardi de Lumière (1977-1991) pour le second -, le texte d'olivier Gleam étudie la manière dont le théâtre musical met en avant le rapport de l'homme à la machine et à la technologie, préfigurant de manière presque prophétique nos addictions actuelles à diverses formes de ce qui est appelé dans le texte nos ippareils, c'est-à-dire ces types d'objets aujourd'hui connectés de près ou de loin au monde du web. Le personnage conçu par Stockhausen Synthi-Fou, mi-homme mi-synthétiseur, rappelle bien cet homme d'aujourd'hui constamment rivé sur sa tablette ou sur son smartphone. Traitant d'une tout autre problématique, l'article de Paula Espinoza se penche quant à lui sur le théâtre musical chilien, sans doute marqué plus que d'autres par l'influence de formes musicothéâtrales populaires. Tiraillé entre le modère brechtien et l'emprise du musical conçu dans une logique de divertissement, ce 
répertoire illumine d'une nouvelle manière l'hybridité inhérente au genre du théâtre musical. Un deuxième article de Pierre Longuenesse, consacré à des ouvrages contemporains issus de la crise de la dramaturgie traversée par notre époque, propose une analyse fine et détaillée de trois spectacles récents: Shun Kin (2008) de Simon McBurney inspiré d'une nouvelle de l'écrivain japonais Jun'ishirô Tanisaki, Le dieu Bonheur d'Alexis Forestier conçu à partir de fragments inachevés de Brecht et de Heiner Müller et enfin Le Maître et Marguerite d'après Boulgakov dans l'adaptation de McBurney. L'article analyse les perspectives d'un théatre post-dramatique, politique et philosophique, relevant de deux utopies poétiques mais dissemblables issues toutes deux de l'éclatement de la représentation. Dans son passionnant texte sur son expérience de chorégraphe pour une compagnie d'acteurs sourds, Lucie Lataste évoque enfin le concept de musique visuelle portée par le rythme et les vibrations nées de l'osmose entre des acteurs communiquant au moyen de la langue des signes, ou entre les acteurs et leur public, décuplant ainsi les sensations des uns et des autres. L'article conclusif de Muriel Plana, s'il ne se consacre à l'une ou l'autre pièce du répertoire, se donne à lire comme un aperçu synthétique de l'état de l'art, des diverses problématiques inhérentes au genre et des pistes théoriques et épistémologiques qu'il reste encore à explorer. Il brasse notamment les questions liées aux différentes formes du dialogisme évoquées dans la plupart des artistes précédents.

4 S'il est un trait saillant dans l'ensemble de ce volume, c'est bien la question du dialogisme et le refus de considérer le texte et la musique comme des éléments fusionnés. Fidèles aux principes fondateurs de Brecht, dont l'ombre tutélaire plane sur la quasi-totalité des textes réunis ici, le théâtre musical prône la séparation de ses éléments (esthétiques et politiques, littéraires et musicaux...) et sans doute est-ce la spécificité de ce genre si particulier que de maintenir le fait que chacun des arts qui le constituent tienne son propre discours. On note également d'un article à l'autre l'omniprésence du politique, dans le sens retenu par Muriel Plana de «philosophique, critique, expérimental, dialogique et utopico-fantasmatique »(p.215). Dans l'attente de nouveaux travaux sur la question, on se régalera de tous ces regards pluridisciplinaires portés par une équipe de chercheur issue de la musique et de la musicologie, des arts du spectacle et de la pratique théâtrale, de la philosophie, de la littérature et de la littérature comparée, des langues, cultures et civilisations étrangères. Il eût été paradoxal qu'un tel objet ne fût pas examiné par des regards croisés et complémentaires, même si l'on ne peut que constater d'un texte à l'autre une évidente convergence des différents points de vue.

INDEX

Keywords : musical, musical theatre, opera

Mots-clés : comédie musicale, opéra, théâtre musical 
AUTEURS

PIERRE DEGOTT

Professeur

Université de Lorraine

pierre.degott@univ-lorraine.fr 